\title{
Real-time trajectory generation for sensor-guided robotic laser welding
}

em

Menno de Graaf, Ronald Aarts, Ben Jonker

University of Twente, Laboratory of Mechanical Automation Institute of Mechanics, Processes and Control - Twente

P.O. Box 217, 7500 AE Enschede, The Netherlands

mpact

+31-(0)53-4895442/2502, m.w.degraaf@utwente.nl

\section{Introduction}

Robotic laser welding imposes high demands on the used manipulator as high accuracies (down to $0.1 \mathrm{~mm}$ ) have to be reached at high velocities (up to $250 \mathrm{~mm} / \mathrm{s}$ ). To meet these specifications with industrial robots, a sensor measuring at the robot tip needs to be applied.

\section{Objectives}

Integration of an optical seam-tracking sensor in a robotic laser welding cell for:

- Sensor-guided teaching of the 3D seam trajectory.

- Increasing the positional accuracy during welding.

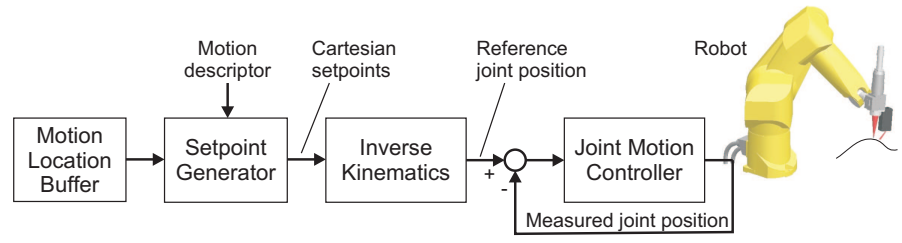

Figure 1 : Real-time control architecture

\section{Methods}

To use sensor information obtained during the robot motion a real-time trajectory generator (Figure 1) based on cubic interpolation of both position and orientation has been implemented on an industrial Stäubli robot. It calculates setpoints for the robot joint motion controller every $4 \mathrm{~ms}$. Features of the trajectory generator are:

- The setpoints are calculated on-the-fly, which allows the addition of cartesian locations to the Motion Location Buffer during the robot motion.

- Seam locations obtained from the robot and seamtracking sensor can be added to the Motion Location Buffer after proper filtering. Such seam locations are e.g. computed from synchronised data of robot joint angles and sensor measurement

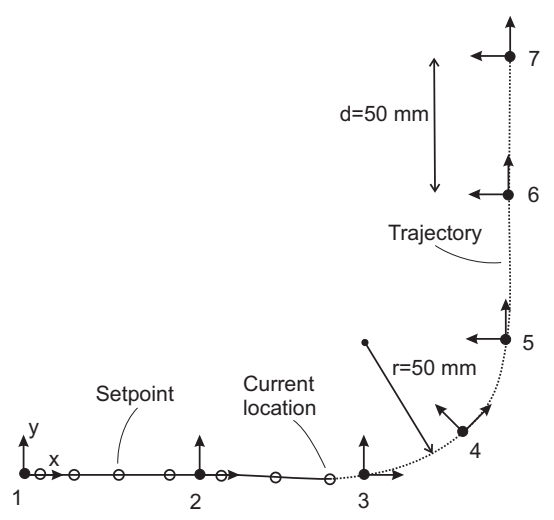

Figure 2 : Corner trajectory

\section{Results}

A corner trajectory (Figure 2) has been replayed with a maximum linear velocity of $100 \mathrm{~mm} / \mathrm{s}$ and rotational velocity of $60 \mathrm{deg} / \mathrm{s}$.
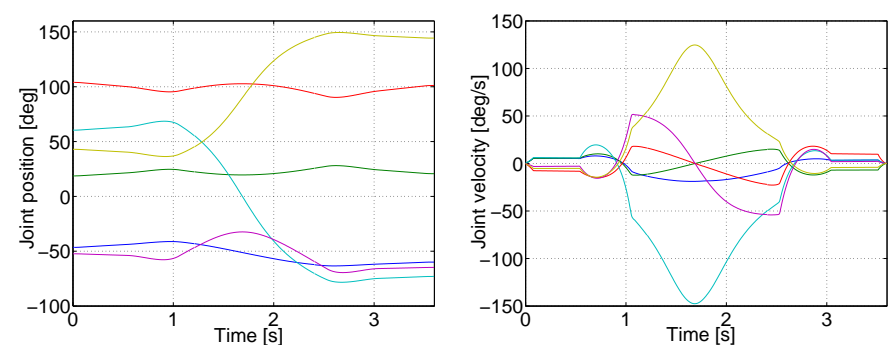

Figure 3 : Reference joint position and velocity (Joint 1, Joint 2, Joint 3, Joint 4, Joint 5, Joint 6). Both the reference joint position and velocity are smooth.
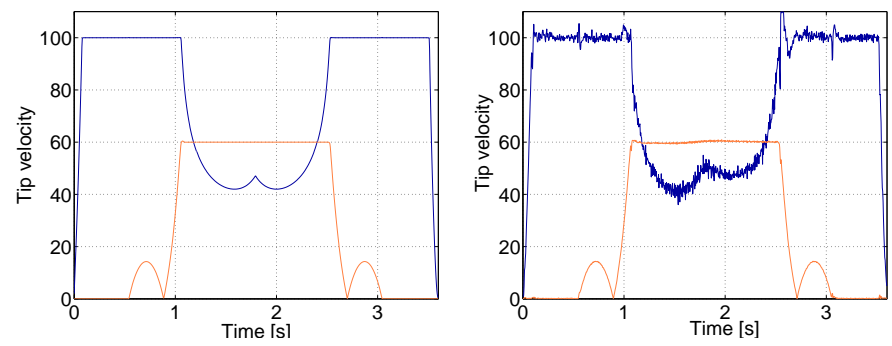

Figure 4 : Reference and measured tip velocity (Linear $[\mathrm{mm} / \mathrm{s}]$, Rotational $[\mathrm{deg} / \mathrm{s}])$. The linear velocity decreases at the corner, because of the bounds in rotational velocity.

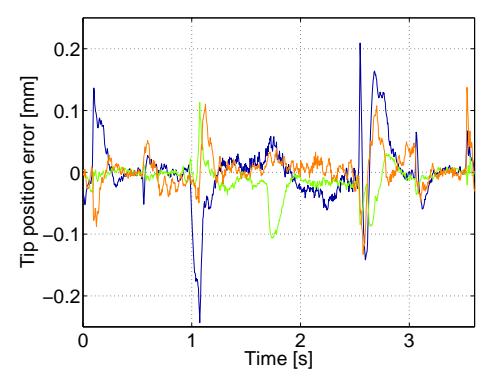

Figure 5 : Measured tip position error $(X, Y, Z)$ based on joint measurements. The tip position error is largest at the acceleration/deceleration phases and when the joint velocity changes sign (joint friction).

\section{References}

1. M.W. de Graaf et al, Real-time trajectory generation for sensorguided robotic lase welding, Submitted to the IFAC Syroco 2006 conference, Bologna, 2006 